\title{
An Effective Solution to Eliminate DC-Offset for Extracting the Phase and Frequency of Grid Voltage
}

\author{
Fehmi Sevilmiş (iD) and Hulusi Karaca $\mathbb{D}$ \\ Department of Electrical and Electronics Engineering, Selçuk University, Konya 42075, Turkey \\ Correspondence should be addressed to Fehmi Sevilmiş; fehmisevilmis@selcuk.edu.tr
}

Received 25 April 2021; Revised 27 August 2021; Accepted 9 September 2021; Published 23 September 2021

Academic Editor: Giuseppe Fedele

Copyright (C) 2021 Fehmi Sevilmiş and Hulusi Karaca. This is an open access article distributed under the Creative Commons Attribution License, which permits unrestricted use, distribution, and reproduction in any medium, provided the original work is properly cited.

\begin{abstract}
Recently, several approaches with the ability to reject the DC-offset in phase locked loop (PLL) methods have been developed. These approaches include different filtering structures which can be classified into two categories: prefiltering before the PLL input and in-loop filtering in the PLL control loop. As highlighted in the literature, the DC-offset rejection methods based on in-loop filtering have received less attention due to their slow dynamic performance. Therefore, this paper proposes an alternative DCoffset rejection technique as in-loop filtering of the PLL. The effectiveness of the proposed PLL is confirmed by simulation and experimental results.
\end{abstract}

\section{Introduction}

Rapid and precise extraction of grid voltage frequency and phase angle is a critical process for grid interactive converters and microgrids $[1,2]$. According to grid codes, to ensure frequency and voltage support to the grid when faults emerge, interconnected renewable energy sources (RES) need fault ride through (FRT) ability. In practice, fast and smoothly tracking of the grid phase angle is specially wanted under frequency and phase variations, various short-term disturbances, voltage sags, DC-offset, and so on. So, research studies on how to develop the performance of PLLs connected to faulty grids without violating the grid code limits are a topic of maximum interest [3-6].

Synchronous reference frame-phase locked loop (SRFPLL) is broadly used thanks to advantages of its fast dynamic performance and simple structure $[7,8]$. However, the performance of SRF-PLL is dramatically deteriorated in case of existence of DC-offset on grid voltages. The DC-offset component may occur due to measurement devices, DC injection from generation systems, A/D conversion processes, grid faults, and so on [9]. This component causes fundamental frequency fluctuations in phase angle and frequency measurement. To overcome this problem, several techniques have been suggested in the literature.

In [10], a band-pass filter (BPF) is introduced in the prefiltering stage of the PLL to eliminate the DC-offset component. Although the BPF can completely remove the DC-offset, it significantly reduces the dynamic performance of the PLL. In [11], an adaptive first-order low-pass filter (LPF) is proposed at the PLL input. In this DC-offset removal method based on the study of [12], the grid voltages passed through LPF are subtracted from their original input voltages. This method is a simple approach to destroy the DC-offset; however, it may deteriorate the harmonic filtering capability of the system. In [13], five DC-offset removal techniques based on prefiltering and in-loop filtering are analyzed in detail. Among these methods, the cross-feedback network, complex-coefficient filter, and $\alpha \beta$-frame delayed signal cancellation (DSC) operator are used in prefilter stage of PLL while the notch filter (NF) and $d q$-frame DSC ( $d q D S C)$ operator are used in its in-loop filter stage. It is reported in [13] that the PLLs based on in-loop filtering effectively eliminate the DC-offset; however, they cause longer transient response time compared with the PLLs based on prefiltering. Some other PLLs designed to block the DC-offset can be accessed in [14-16]. 
The objective of presented work is to develop the performance of the PLL caused by in-loop filtering in the presence of DC-offset. To minimize the transient response time, all-pass filter (APF)-based DSC operator ( $d q \mathrm{ADSC}$ $\mathrm{PLL}$ ) is proposed. In addition to poor dynamic performance of the standard $d q \mathrm{DSC}-\mathrm{PLL}$, another drawback of the $d q$ DSC-PLL is that it has a discretization error in case of grid frequency variation even if the DSC operator is adaptive [17]. Thanks to the APF-based DSC operator, the discretization error that occurs in the standard $d q \mathrm{DSC}-\mathrm{PLL}$ is eliminated in the proposed $d q$ ADSC-PLL.

In brief, the main contributions of the proposed PLL can be listed as follows:

(1) The suggested $d q$ ADSC-PLL has a simple structure and is easy to implement

(2) Its parameter design process is straightforward because only one parameter has to be set in its structure

(3) It considerably reduces the convergence time

(4) In addition to effectively blocking the DC-offset component, it minimizes the discretization errors

The effectiveness and feasibility of the suggested method are validated by simulation and experimental studies and comparison with standard $d q$ DSC-PLL and NF-PLL, which have the different filters in the loop of the PLL to cancel out the DC-offset disturbance component.

\section{Description of $\boldsymbol{d q D S C - P L L}$ and NF-PLL}

2.1. $d q D S C-P L L$. Figure 1 presents the topology of classical $d q \mathrm{DSC}-\mathrm{PLL}$. In the $d q \mathrm{DSC}-\mathrm{PLL}$, firstly, three-phase grid signals $\left(V_{a}, V_{b}\right.$, and $\left.V_{c}\right)$ are measured. Then, these signals are converted into synchronous reference frame components $\left(V_{d}\right.$ and $V_{q}$ ). The $d q$ DSC-PLL has the DSC operator in PLL control loop. It also has a magnitude normalization processing after the DSC operator. Thus, the PLL control loop becomes independent against the grid voltage amplitude changes.

The DSC operator is a kind of finite impulse response (FIR) filter. The transfer function of the $d q \mathrm{DSC}$ operator is defined as follows [17]:

$$
d q \operatorname{DSC}_{n}(s)=\frac{1+e^{(-T / n) s}}{2}
$$

where $n$ is a delay factor and $T$ is the fundamental cycle of grid voltages.

$n$ is chosen as 2 to block the DC-offset component. Substituting $n=2$ and $s=j \omega$ into (1), the frequency response of $d q \mathrm{DSC}_{2}$ can be obtained as

$$
d q \operatorname{DSC}_{2}(j \omega)=\left|\cos \left(\frac{\omega T}{4}\right)\right| \angle-\left(\frac{\omega T}{4}\right) .
$$

2.2. NF-PLL. Figure 2 demonstrates the block structure of the standard NF-PLL. Here, the measured grid signals are converted to $d q$-frame variables. The NF-PLL has a secondorder notch filter as in-loop filtering of the PLL. As in

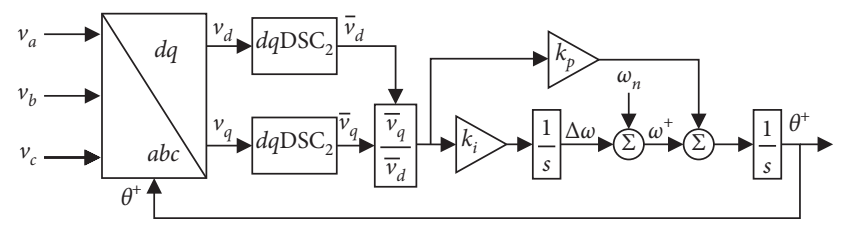

Figure 1: Structure of the standard $d q$ DSC-PLL.

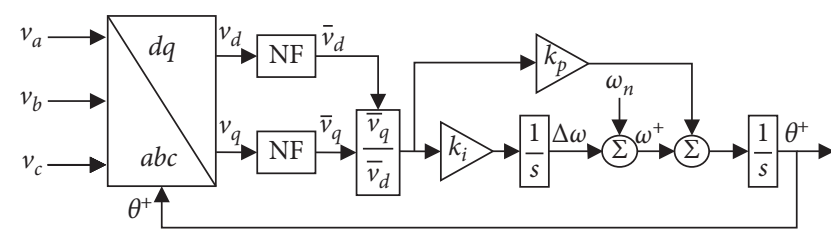

FIGURE 2: NF-PLL's block structure.

$d q$ DSC-PLL, the NF-PLL also has an amplitude normalization processing. Actually, the design of the NF-PLL is based on the replacement of the DSC operator in the $d q$ DSCPLL structure with a notch filter as illustrated in Figure 2.

The NF which acts as a band rejection filter eliminates frequencies within a restricted band. It also passes other frequencies without change. The transfer function of secondorder NF is expressed as

$$
\mathrm{NF}(s)=\frac{s^{2}+\omega_{n f}^{2}}{s^{2}+\left(\omega_{n f} / Q\right) s+\omega_{n f}^{2}},
$$

where $Q$ and $\omega_{n f}$ indicate the quality factor and notch frequency of NF, respectively.

It is recommended in [13] to set the $\omega_{n f}$ as $2 \pi 50 \mathrm{rad} / \mathrm{s}$ (notice that the NF is nonadaptive) for the $50 \mathrm{~Hz}$ system because DC-offset component converts to fundamental frequency disturbance component after Park transformation. The $Q$ value directly affects the bandwidth of the filter. In case of large changes in the grid frequency, selecting wide bandwidth allows the DC-offset to be effectively destroyed. Therefore, it should be chosen as 0.707 [13]. However, the wide bandwidth causes dynamic performance of PLL to slow down.

\section{Proposed $d q A D S C-P L L$}

3.1. Description. Figure 3 illustrates the topology of suggested $d q \mathrm{ADSC}-\mathrm{PLL}$, in which the standard DSC operator is replaced by the APF-based DSC operator in $d q$-frame. In this study, APF is preferred as a loop filter because it has many advantages such as low computational load, simple digital implementation, and fast transient response [18, 19].

The APF has a low-pass filter feature. Transfer function of a first-order APF can be expressed as

$$
\operatorname{APF}(s)=\frac{\omega_{n}-s}{\omega_{n}+s},
$$

where $\omega_{n}$ is the nominal grid frequency.

Substituting $s=j \omega$ into (4), the gain and phase responses of the APF are determined as 


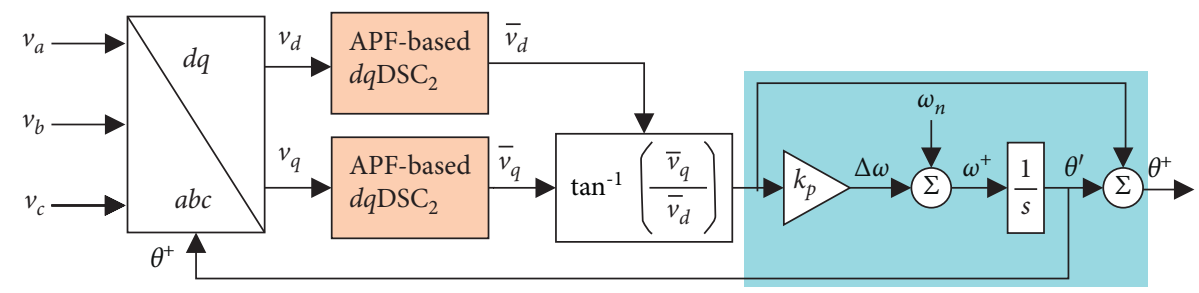

FIgURE 3: Structure of suggested $d q$ ADSC-PLL.

$$
\operatorname{APF}(j \omega)=1 \angle-2 \arctan \left(\frac{\omega}{\omega_{n}}\right) .
$$

In Figure 4, the Bode graph of the APF is shown. From the figure, the APF for all frequencies ensures unity gain (e.g., the APF does not change the amplitude of frequencies) and solely varies the input signal phase. When the obtained grid frequency equals the nominal frequency (where $\omega_{n}=2 \pi 50 \mathrm{rad} / \mathrm{s}$ ), a $90^{\circ}$ phase shift happens at the nominal frequency [19]. The structure of APF can be simply designed as in Figure 5. Here, $q v$ is a $90^{\circ}$ lagging-phase of input signal (v).

In the proposed APF-based $d q \mathrm{DSC}_{2}$ operator, two APF modules are cascaded to eliminate the DC-offset (see Figure 6). In this way, a $180^{\circ}$ phase shift is acquired by means of the cascaded two APFs. The transfer function of the cascaded APF (CAPF) module is obtained as

$$
\operatorname{CAPF}(s)=\frac{s^{2}-2 \omega_{n} s+\omega_{n}^{2}}{s^{2}+2 \omega_{n} s+\omega_{n}^{2}} .
$$

As observed from Figure 6, the transfer function of the APF-based $d q \mathrm{DSC}_{2}$ operator is easily expressed as follows:

$$
d q \operatorname{ADSC}_{2}(s)=\frac{s^{2}+\omega_{n}^{2}}{s^{2}+2 \omega_{n} s+\omega_{n}^{2}} .
$$

Figure 7 shows the Bode plot of the proposed $d q \mathrm{ADSC}_{2}$ operator in $d q$-frame. While the fundamental frequency positive sequence (FFPS) transforms into DC component, DC-offset turns to fundamental frequency disturbance component after Park transformation. It can be seen from Figure 7 that the suggested operator ensures unity gain at the nominal grid frequency $(0 \mathrm{~Hz})$ and zero gain at $-50 \mathrm{~Hz}$ in $d q$-frame. That is, the proposed $d q \mathrm{ADSC}_{2}$ operator rejects the DC-offset and passes the FFPS component completely.

In the proposed method, the controller design is different from that of the standard $d q$ DSC-PLL and NF-PLL. As can be seen from the standard $d q$ DSC-PLL and NF-PLL structures given in Figures 1 and 2, respectively, the conventional PI controllers are used in the control loop of these PLLs. However, as seen in Figure 3, PI is not used in the controlling stage of the proposed $d q \mathrm{ADSC}-\mathrm{PLL}$ method. Instead of the PI controller, the controller pattern in [20] is preferred. In this controller structure, there is only a simple gain $\left(k_{p}\right)$. Besides, this structure has a path to phase output. Thus, it has two poles at the origin, as given in (10). Although this controller pattern seems as a type- 1 system, it is actually type-2 system. And it has two major advantages: (1) it
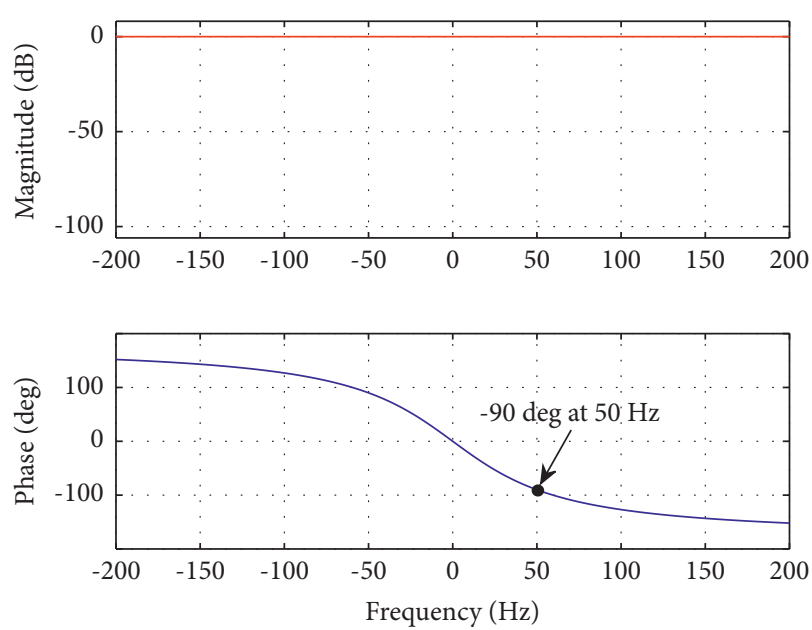

Figure 4: Bode plot of APF.

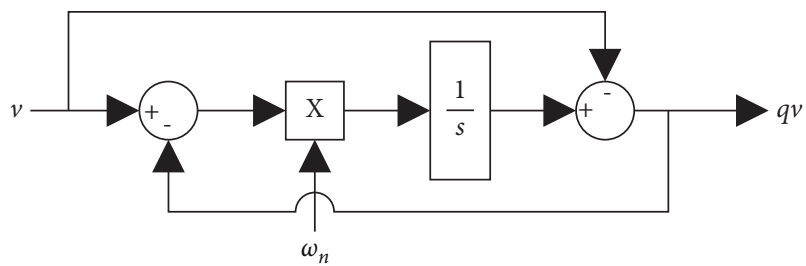

Figure 5: Structure of APF.

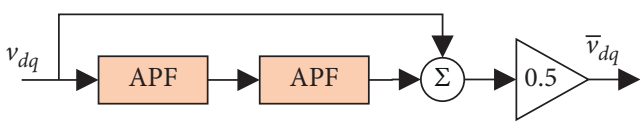

Figure 6: Structure of APF-based $d q \mathrm{DSC}_{2}$.

increases dynamic performance significantly and (2) it provides a simpler controller parameter design procedure, which is discussed in the later section in detail.

3.2. Discretization of the Filters. Filters must be expressed in a discrete time domain in order to be used in practical applications. To discrete the APF given in continuous time domain as illustrated in Figure 5, the third-order Adams-Bashforth method [21] is preferred in this paper. The reason for using this method is that it provides high accuracy in discretization process. The integrator in the APF is approximated by 

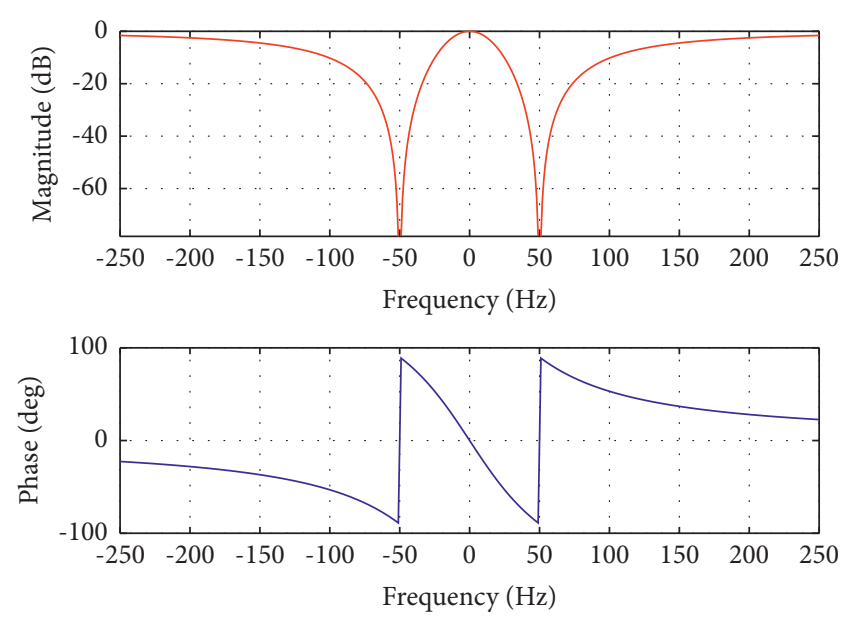

Figure 7: Bode plot of the proposed APF-based $d q \mathrm{DSC}_{2}$.

$$
\frac{1}{s} \Longleftrightarrow \frac{T_{s}}{12} \frac{23 z^{-1}-16 z^{-2}+5 z^{-3}}{1-z^{-1}}
$$

where $T_{s}$ denotes the sampling time. In other words, it is sufficient to replace the integrator in Figure 5 with the discrete form in (8) to discrete the APF.

Similar to APF discretization, the approximate equation in (8) is considered for integrators in NF structure to discrete the NF.

In order to discrete the standard $d q \mathrm{DSC}_{2}$ given in (1), the block structure in Figure 8 is used. In Figure $8, N$ is defined as $T /\left(n T_{s}\right) . N$ is also an integer value. Here, $T_{s}$ is the sampling time, $n$ is a delay factor, and $T$ is the grid period. If grid frequency is equal to $50 \mathrm{~Hz}, n$ is chosen as 2 to reject the DC-offset, and sampling frequency is set to be $10 \mathrm{kHz}$; $N$ equals 100 . When frequency variation occurs besides the DC-offset and the DSC operator is adaptive, $N$ is calculated as a noninteger value. Therefore, $N$ is rounded to the integer value. This situation causes the discretization error. Figure 9 shows the phase errors of the standard $d q$ DSC-PLL and proposed $d q \mathrm{ADSC}-\mathrm{PLL}$ in presence of DC-offset with a $+2 \mathrm{~Hz}$ frequency jump. Notice that the DSC operator in the standard $d q$ DSC-PLL and the CAPF module in the proposed $d q$ ADSC-PLL are adaptive. It is observed from Figure 9 that the discretization error occurs on estimated phase angle in the standard $d q$ DSC-PLL. This error is caused by rounding the $N$ value to the nearest integer value. Since the proposed PLL uses APF instead of the DSC operator, there is no such a problem in the proposed PLL. This is another contribution of this paper to the literature.

\subsection{Parameter Design Guideline of Proposed dqADSC-PLL.} In the proposed $d q \mathrm{ADSC}-\mathrm{PLL}, k_{p}$ is the only one parameter to be designed. In order to design $k_{p}$, as shown in Figure 10, the small-signal model of the $d q$ ADSC-PLL should be derived. As shown in (7), the second-order terms of the proposed $d q \mathrm{ADSC}_{2}$ operator in the low frequency range can be neglected as follows:

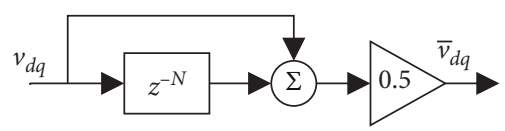

FIgURE 8: Discrete time implementation of $d q \mathrm{DSC}_{2}$.

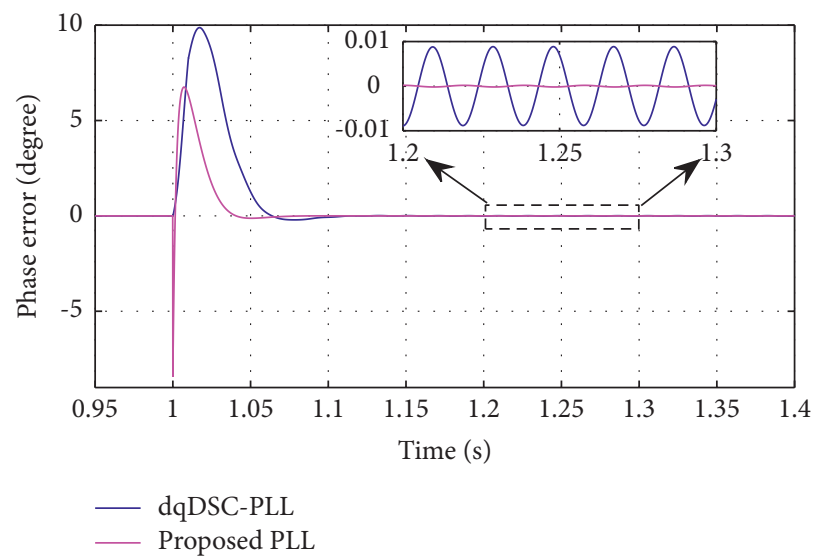

FIgURE 9: Discretization error in presence of DC-offset with a $+2 \mathrm{~Hz}$ frequency step change.

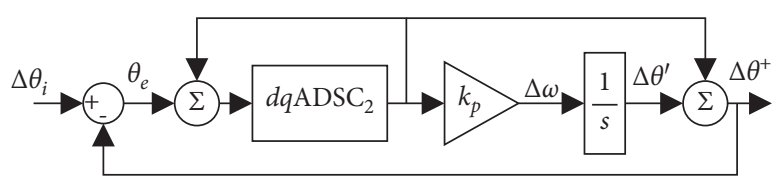

FIGURE 10: Small-signal model of the $d q$ ADSC-PLL.

$d q \operatorname{ADSC}_{2}(s)=\frac{s^{2}+\omega_{n}^{2}}{s^{2}+2 \omega_{n} s+\omega_{n}^{2}} \approx \frac{\omega_{n}^{2}}{2 \omega_{n} s+\omega_{n}^{2}}=\frac{\omega_{n} / 2}{s+\left(\omega_{n} / 2\right)}$

As understood from (9), the approximate transfer function of $d q \mathrm{ADSC}_{2}$ is equivalent to a first-order low-pass filter, in which its cut-off frequency is $\omega_{n} / 2$.

The open-loop transfer function of the $d q$ ADSC-PLL by using the small-signal model is expressed as

$$
\begin{aligned}
G_{\mathrm{ol}}(s) & =\frac{\Delta \theta^{+}(s)}{\theta_{e}}=\left(\frac{d q \operatorname{ADSC}_{2}(s)}{1-d q \operatorname{ADSC}_{2}(s)}\right)\left(1+\frac{k_{p}}{s}\right) \\
& =\frac{\left(\omega_{n} / 2\right)\left(s+k_{p}\right)}{s^{2}} .
\end{aligned}
$$

As can be understood from (10), the open-loop transfer function of the suggested method has two poles at the origin, which means it turns into a type-2 system.

Using (10), the transfer function of phase error can be obtained as

$$
G_{e}(s)=\frac{\theta_{e}}{\Delta \theta_{i}(s)}=\left(\frac{1}{1+G_{\mathrm{ol}}(s)}\right)
$$

Using (11), the variation of the $2 \%$ settling time of the $d q \mathrm{ADSC}-\mathrm{PLL}$ under phase and frequency jump conditions, as shown in Figure 11, can be attained as a function of $k_{p}$. It is 


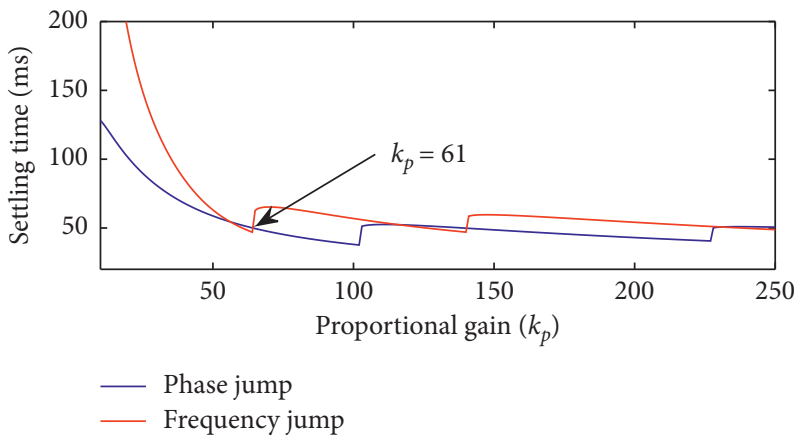

Figure 11: $2 \%$ settling time of $d q$ ADSC-PLL as a function of $k_{p}$ under phase and frequency jump.

shown that $k_{p}$ of the $d q \mathrm{ADSC}-\mathrm{PLL}$ is chosen as 61 , which is the best value under both conditions.

Finally, the performance of the proposed $d q$ ADSC-PLL and its model is compared to judge the accuracy of smallsignal model under frequency and phase jump conditions. Figure 12 shows that its model predicts the dynamic behaviour of $d q \mathrm{ADSC}$-PLL with high accuracy.

3.4. Stability Analysis. To analyze the stability of the method, its model can be used. Based on the model given in Figure 10, the closed-loop transfer function of the proposed $d q \mathrm{ADSC}$ PLL is determined as follows:

$$
G_{\mathrm{cl}}(s)=\frac{\Delta \theta^{+}(s)}{\Delta \theta_{i}(s)}=\frac{\left(\omega_{n} / 2\right)\left(s+k_{p}\right)}{s^{2}+\left(\omega_{n} / 2\right) s+\left(\omega_{n} / 2\right) k_{p}} .
$$

Considering the characteristic equation (12), the Routh-Hurwitz criterion is used for stability analysis of the system. According to this criterion, the suggested $d q$ ADSCPLL is stable for $\omega_{n}>0$ and $k_{p}>0$.

\section{Simulation and Experimental Results}

The effectiveness of suggested $d q$ ADSC-PLL is validated by simulation and experimental studies. The simulation studies are realized in Matlab/Simulink software. The experimental studies are performed by a TMS320F28335 DSP. Sampling frequency, magnitude of the grid voltages, and nominal grid frequency $\left(\omega_{n}\right)$ are tuned to $10 \mathrm{kHz}, 1$ p.u., and $2 \pi 50 \mathrm{rad} / \mathrm{s}$, respectively. Moreover, the standard $d q \mathrm{DSC}-\mathrm{PLL}$ and NFPLL [13] are carried out to compare their performance with the proposed $d q \mathrm{ADSC}$-PLL. In the experiments, the grid voltages are created internally in the DSP. All PLLs are employed in the DSP. To demonstrate the grid voltages, phase error, and estimated frequency on the oscilloscope, these signals are passed through external low-pass filter. Figure 13 shows the experimental setup.

The control parameter $\left(k_{p}\right)$ of the proposed PLL is obtained as 61 in the previous section. The parameters of the standard $d q$ DSC-PLL and NF-PLL are chosen as in [13] (e.g., $k_{p}=82.84$ and $k_{i}=2842.7$ for $d q$ DSC-PLL; $k_{p}=92$, and $k_{i}=3507.1$ for NF-PLL).

To compare the DC-offset rejection methods under different grid scenarios, four test conditions are considered as follows: (i) Test 1. The frequency jumps from $50 \mathrm{~Hz}$ to $52 \mathrm{~Hz}$ suddenly.

(ii) Test 2. The grid signals subject a phase jump of $+40^{\circ}$. The frequency is set at $50 \mathrm{~Hz}$.

(iii) Test 3. The DC-offset components are added to all phases. The values of these components are $0.2,0.1$, and -0.2 p.u. for phases $A, B$, and $C$, respectively. The grid frequency is tuned as $50 \mathrm{~Hz}$.

(iv) Test 4 . The same DC-offsets are added to each phase as in the test 3 . However, the frequency is tuned as $52 \mathrm{~Hz}$ simultaneously. Notice that all PLLs are adaptive during this test.

Figure 14 demonstrates the estimated frequency results for the first test. As shown, the suggested $d q$ ADSC-PLL presents the fast dynamic response. Its settling time is about 1.85 periods. The settling times of the standard $d q$ DSC-PLL and NF-PLL are about 2.9 periods and 2.6 periods, respectively.

Figure 15 demonstrates the phase error results for the second test. It is shown that the suggested $d q$ ADSC-PLL ensures a fast dynamic performance again. The settling time of the proposed PLL (around 2.5 periods) is lower than that of the standard $d q$ DSC-PLL (around 3.6 periods) and NFPLL (around 3.2 periods). The phase-angle phase overshoot is around $8.7 \%, 13.8 \%$, and $14.3 \%$ for the proposed $d q A D S C$ PLL, standard $d q D S C-P L L$, and NF-PLL, respectively. This test demonstrates that the proposed DC-offset rejection method has the best performance from the point of both settling time and phase overshoot.

Figure 16 shows the experimental results of phase error for the third test. It is illustrated that all PLLs in the steadystate have no phase-angle error. However, the suggested PLL offers a better dynamic performance than that of the $d q \mathrm{DSC}$ PLL and NF-PLL. On the other hand, if frequency change occurs besides the DC-offset, phase error occurs in all PLL methods. To overcome such a situation, the DSC operator in the standard $d q \mathrm{DSC}-\mathrm{PLL}$, the NF in the standard NF-PLL, and the CAPF module in the proposed $d q$ ADSC-PLL can be adapted against the frequency changes. Figure 17 illustrates the phase errors of adaptive PLL methods for the fourth test condition. As shown, the proposed $d q$ ADSC-PLL presents a quite fast dynamic performance. Furthermore, the proposed DC-offset rejection method provides a lower phase error overshoot compared with two other PLLs. 


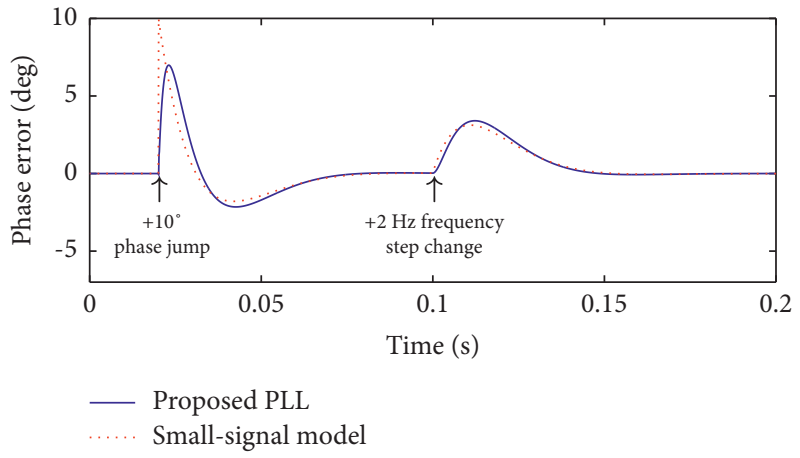

Figure 12: Accuracy of the dqADSC-PLL's model.

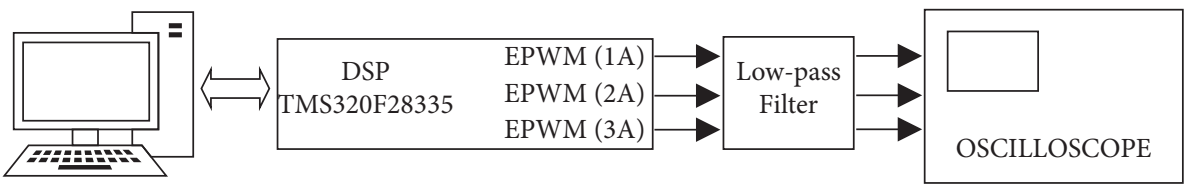

Figure 13: Experimental setup.
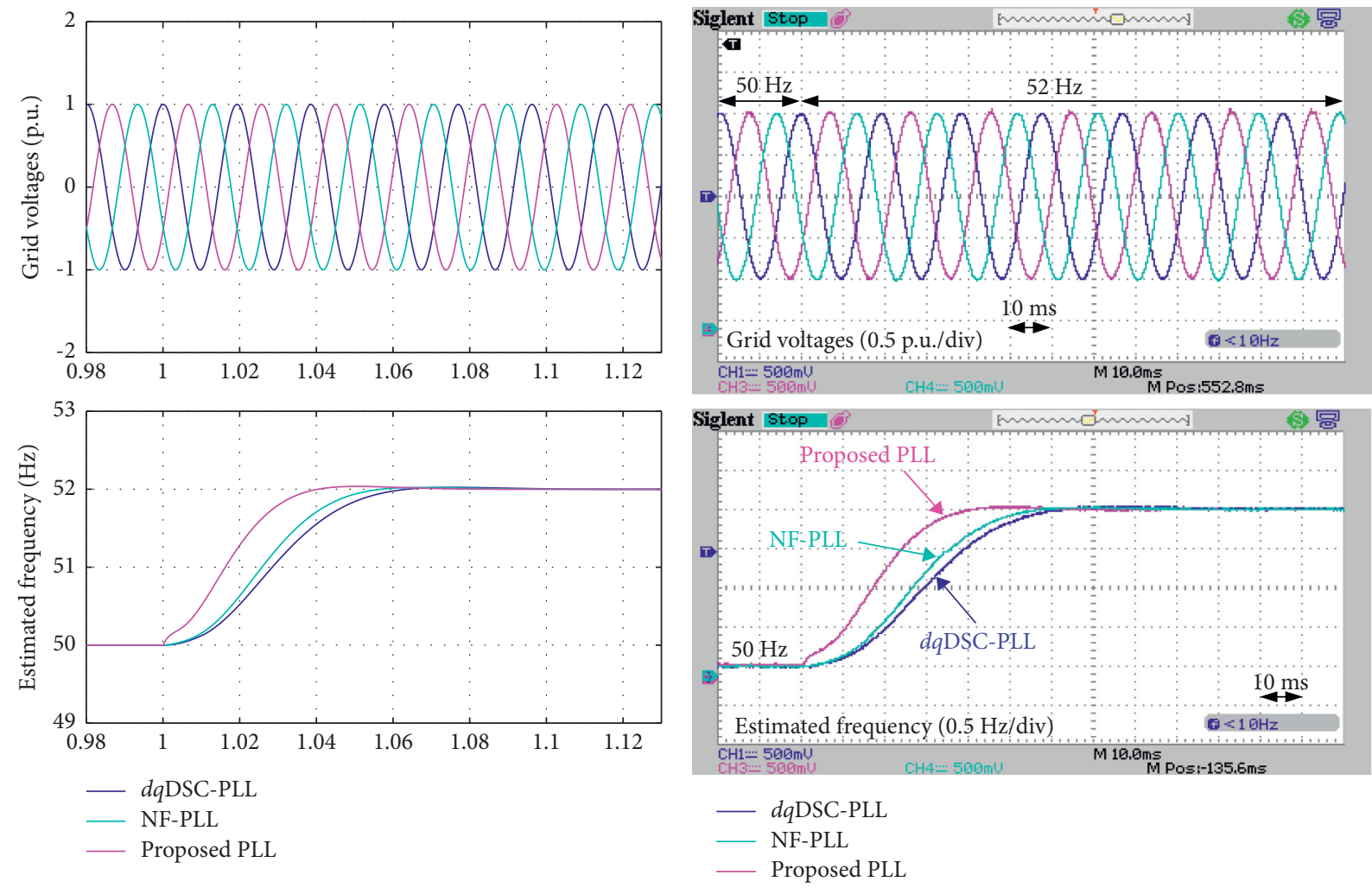

(a)

(b)

FIGURE 14: Frequency jump of $+2 \mathrm{~Hz}$ (Test 1): (a) simulation results; (b) experimental results. 


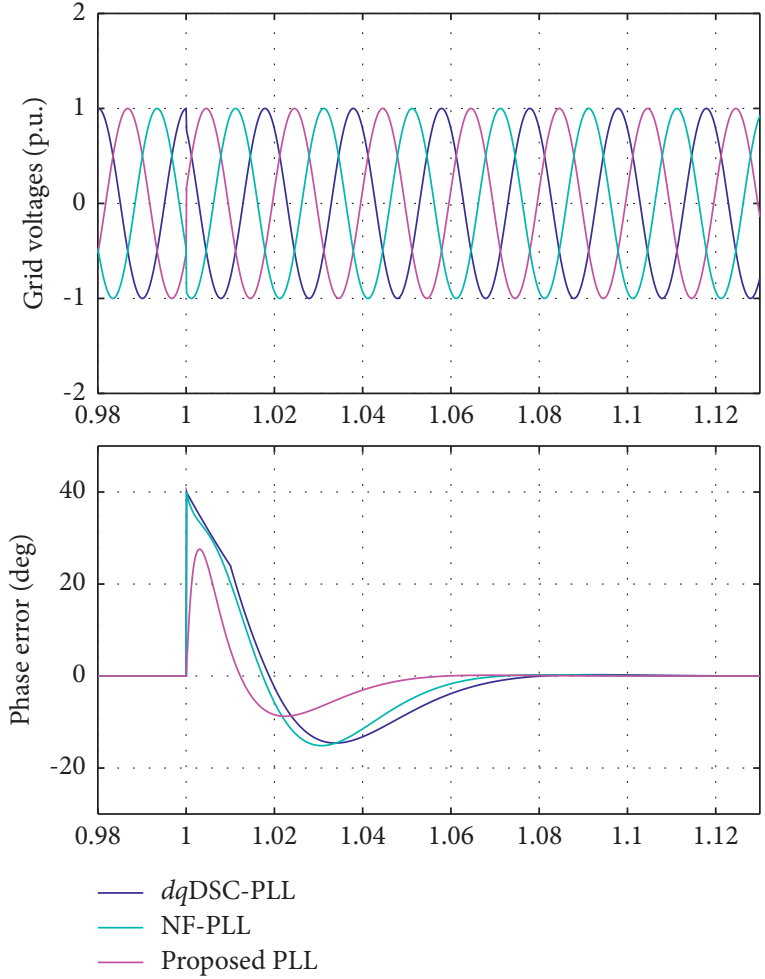

(a)
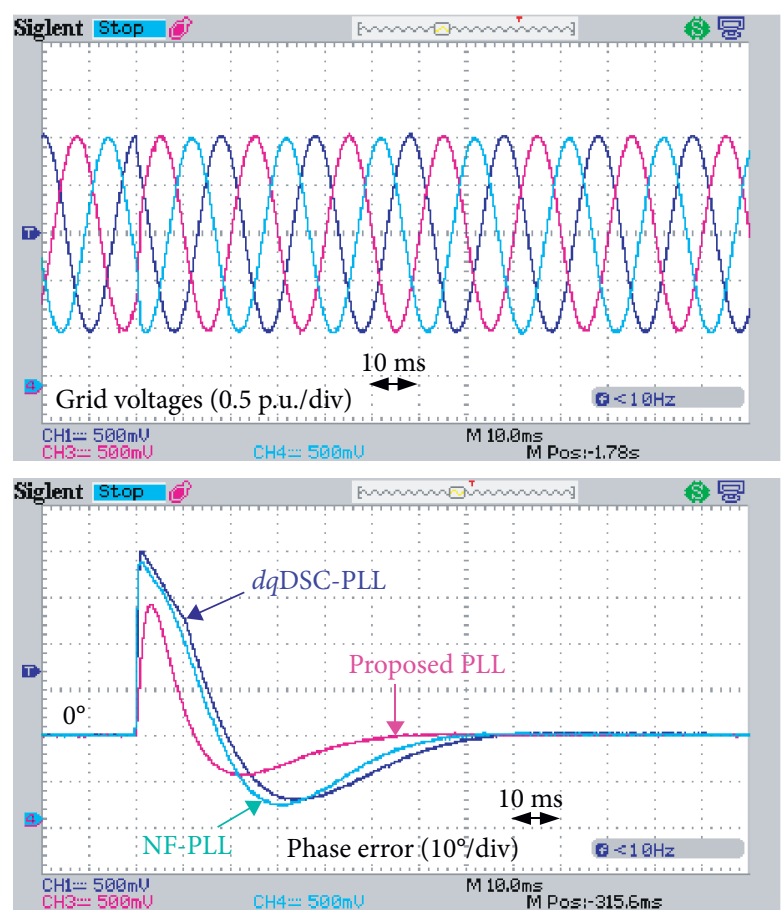

- $d q$ DSC-PLL

- NF-PLL

— Proposed PLL

Figure 15: Phase jump of $+40^{\circ}$ (Test 2): (a) simulation results; (b) experimental results.
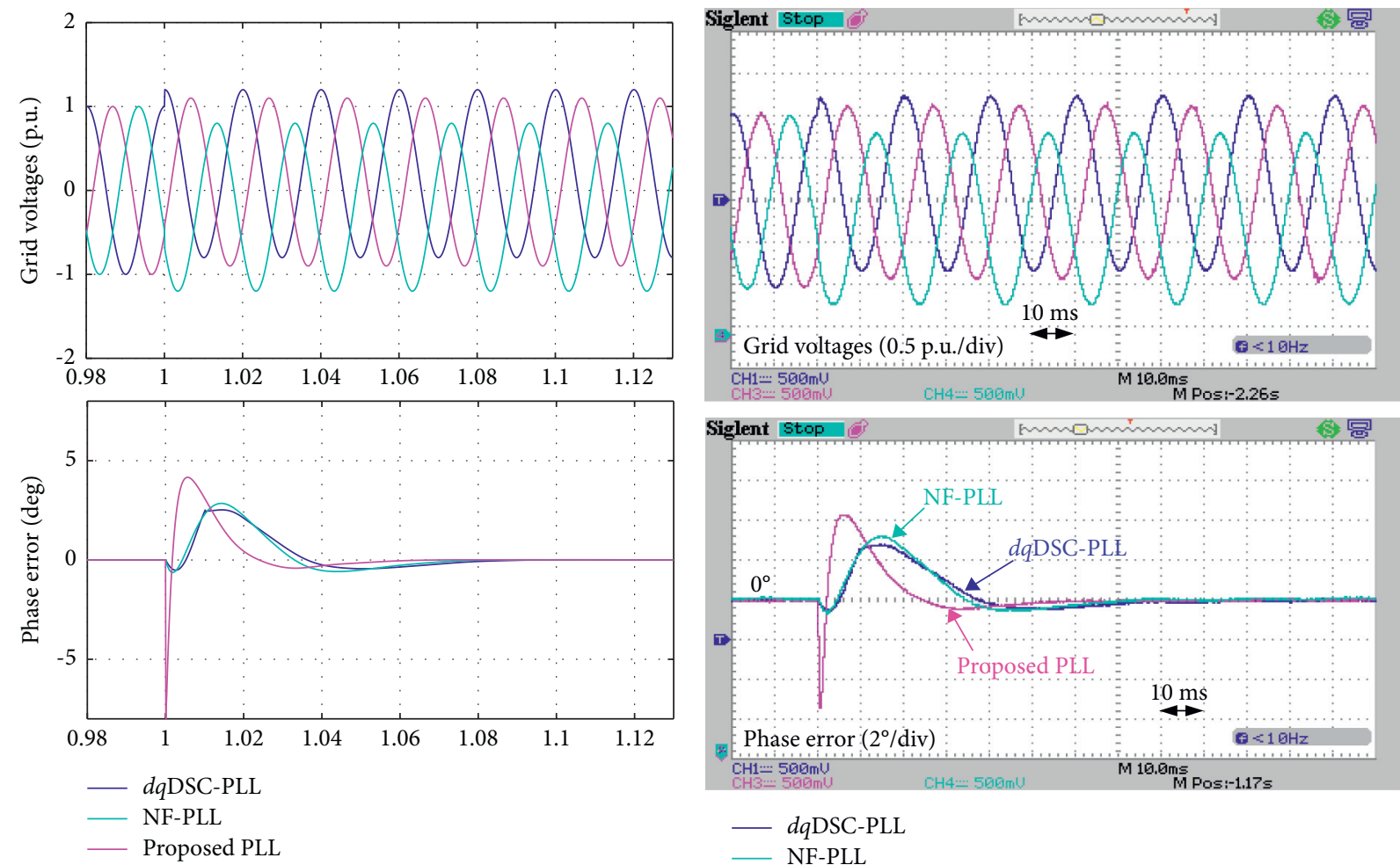

$-d q$ DSC-PLL

NF-PLL

— Proposed PLL

(a)

(b)

Figure 16: DC-offset at $50 \mathrm{~Hz}$ (Test 3): (a) simulation results; (b) experimental results. 

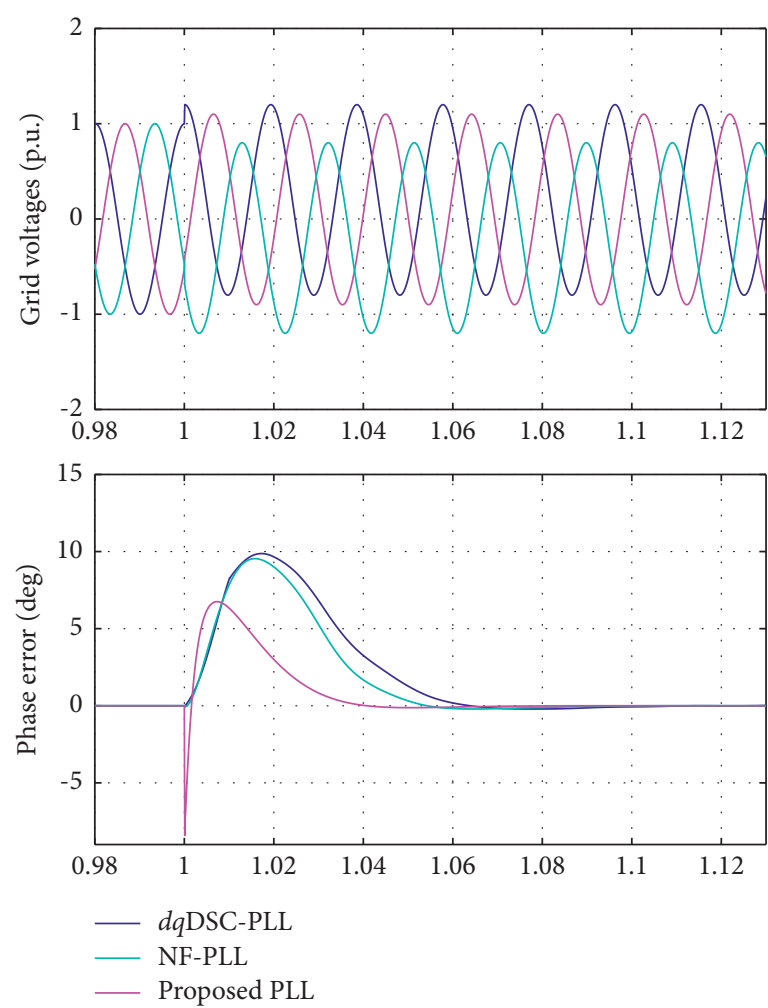

(a)
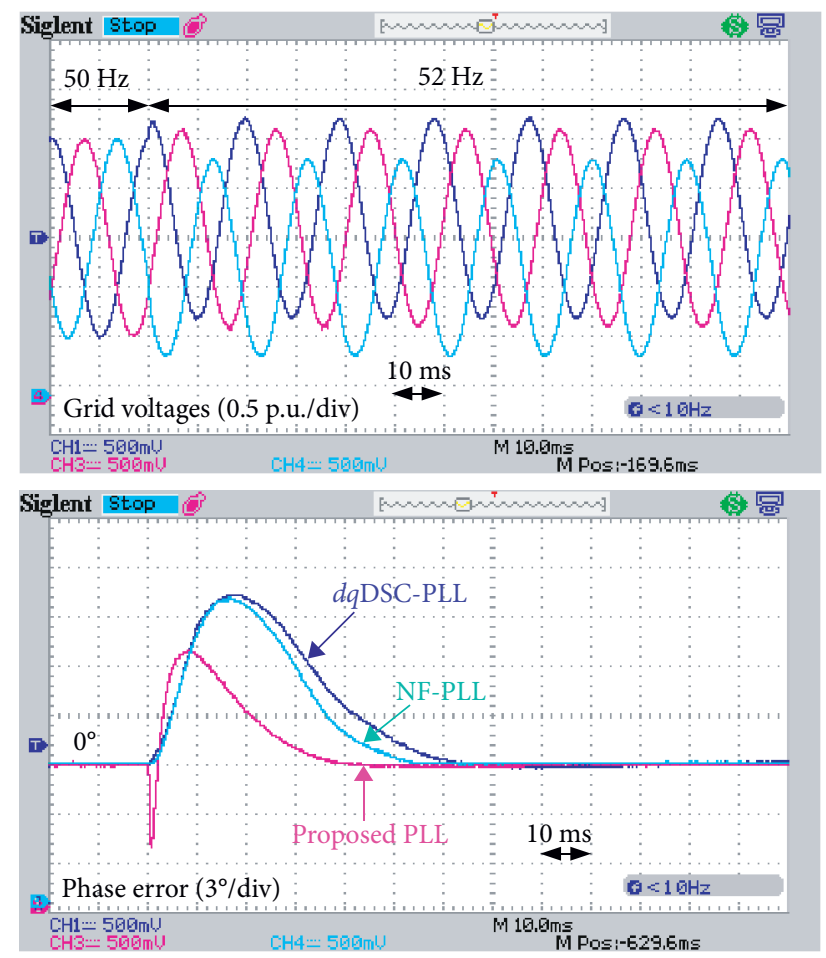

$d q$ DSC-PLL

NF-PLL

— Proposed PLL

FIGURE 17: DC-offset at $52 \mathrm{~Hz}$ (Test 4): (a) simulation results; (b) experimental results.

\section{Conclusions}

In this paper, a highly effective and simple structured DCoffset elimination technique as in-loop filtering of the PLL is proposed. The proposed method called dqADSC-PLL offers a lower settling time than that of the standard $d q$ DSC-PLL and NF-PLL under all test conditions. Furthermore, it eliminates the discretization errors in the standard $d q$ DSC-PLL thanks to the APF. Several numerical results verify the effectiveness of the suggested $d q$ ADSC-PLL.

\section{Data Availability}

The data used to support the findings of this study are included within the article.

\section{Conflicts of Interest}

The authors declare that there are no conflicts of interest regarding the publication of this paper.

\section{Acknowledgments}

This work was supported by the Scientific Research Projects Coordinating Office of Selçuk University (grant no. 19401090).

\section{References}

[1] M. Karimi-Ghartemani, Enhanced Phase-Locked Loop Structures for Power and Energy Applications, Wiley, Hoboken, USA, 2014.

[2] X. Wang, Z. Yang, B. Fan, and W. Xu, "Control strategy of three-phase photovoltaic inverter under low-voltage ridethrough condition," Mathematical Problems in Engineering, vol. 2015, Article ID 790584, 23 pages, 2015.

[3] L. Hadjidemetriou, E. Kyriakides, and F. Blaabjerg, "An adaptive tuning mechanism for phase-locked loop algorithms for faster time performance of interconnected renewable energy sources," IEEE Transactions on Industry Applications, vol. 51, no. 2, pp. 1792-1804, 2015.

[4] Y. F. Wang and Y. W. Li, "Grid synchronization PLL based on cascaded delayed signal cancellation," IEEE Transactions on Power Electronics, vol. 26, no. 7, pp. 1987-1997, 2011.

[5] F. D. Freijedo, A. G. Yepes, Ó. López, A. Vidal, and J. DovalGandoy, "Three-phase PLLs with fast postfault retracking and steady-state rejection of voltage unbalance and harmonics by means of lead compensation," IEEE Transactions on Power Electronics, vol. 26, no. 1, pp. 85-97, 2011.

[6] A. Luna, J. Rocabert, J. Candela et al., "Grid voltage synchronization for distributed generation systems under grid fault conditions," IEEE Transactions on Industry Applications, vol. 51, no. 4, pp. 3414-3425, 2015.

[7] S. Golestan, J. M. Guerrero, and J. C. Vasquez, "Three-phase PLLs: a review of recent advances," IEEE Transactions on Power Electronics, vol. 32, no. 3, pp. 1894-1907, 2017. 
[8] X. Wan, X. Ding, and H. Hu, “An improved active damping method based on single-loop inverter current control for LCL resonance in grid-connected inverters," Mathematical Problems in Engineering, vol. 2021, Article ID 6643223, 11 pages, 2021.

[9] M. Karimi-Ghartemani, S. A. Khajehoddin, P. K. Jain, A. Bakhshai, and M. Mojiri, "Addressing DC component in PLL and notch filter algorithms," IEEE Transactions on Power Electronics, vol. 27, no. 1, pp. 78-86, 2012.

[10] M. Karimi-Ghartemani, S. A. Khajehoddin, P. K. Jain, and A. Bakhshai, "Comparison of two methods for addressing DC component in phase locked loop (PLL) systems," in Proceedings of the IEEE Energy Conversion Congress and Exposition, pp. 3053-3058, IEEE, Phoenix, AZ, USA, September 2011.

[11] P. Kanjiya, V. Khadkikar, and M. S. El Moursi, “Adaptive lowpass filter based DC offset removal technique for three-phase PLLs," IEEE Transactions on Industrial Electronics, vol. 65, no. 11, pp. 9025-9029, 2018.

[12] D. Dong, D. Boroyevich, P. Mattavelli, and I. Cvetkovic, "A high-performance single-phase Phase-Locked-Loop with fast line-voltage amplitude tracking," in Proceedings of the IEEE Applied Power Electronics Conference and Exposition (APEC), pp. 1622-1628, Fort Worth, TX, USA, March 2011.

[13] S. Golestan, J. M. Guerrero, and G. B. Gharehpetian, "Five approaches to deal with problem of DC offset in phase-locked loop algorithms: design considerations and performance evaluations," IEEE Transactions on Power Electronics, vol. 31, no. 1, pp. 648-661, 2016.

[14] S.-H. Hwang, L. Liu, H. Li, and J.-M. Kim, "DC offset error compensation for synchronous reference frame PLL in singlephase grid-connected converters," IEEE Transactions on Power Electronics, vol. 27, no. 8, pp. 3467-3471, 2012.

[15] S. Golestan, J. M. Guerrero, and J. C. Vasquez, "DC-offset rejection in phase-locked loops: a novel approach," IEEE Transactions on Industrial Electronics, vol. 63, no. 8, pp. 4942-4946, 2016.

[16] Y. Li, D. Wang, Y. Ning, and N. Hui, "DC-offset elimination method for grid synchronisation," Electronics Letters, vol. 53, no. 5, pp. 335-337, 2017.

[17] Y. F. Wang and Y. Wei Li, "Analysis and digital implementation of cascaded delayed-signal-cancellation PLL," IEEE Transactions on Power Electronics, vol. 26, no. 4, pp. 1067-1080, 2011.

[18] S. Beheshtaein, M. Savaghebi, R. M. Cuzner, S. Golestan, and J. M. Guerrero, "Modified secondary-control-based fault current limiter for inverters," IEEE Transactions on Industrial Electronics, vol. 66, no. 6, pp. 4798-4804, 2019.

[19] F. Sevilmiş and H. Karaca, "A fast hybrid PLL with an adaptive all-pass filter under abnormal grid conditions," Electric Power Systems Research, vol. 184, pp. 1-10, 2020.

[20] S. Golestan, F. D. Freijedo, A. Vidal, J. M. Guerrero, and J. Doval-Gandoy, "A quasi-type-1 phase-locked loop structure," IEEE Transactions on Power Electronics, vol. 29, no. 12, pp. 6264-6270, 2014.

[21] M. Ciobotaru, R. Teodorescu, and F. Blaabjerg, "A new singlephase PLL structure based on second order generalized integrator," in Proceedings of the IEEE Power Electronics Specialists Conference, pp. 1-6, IEEE, Jeju, South Korea, June 2006. 